\title{
Supramolecular GAG-like Self-Assembled Glycopeptide Nanofibers Induce Chondrogenesis and Cartilage Regeneration
}

\author{
Seher Ustun Yaylaci, ${ }^{\perp, \dagger}$ Melis Sardan Ekiz, ${ }^{\perp, \dagger}$ Elif Arslan, ${ }^{\dagger}$ Nuray Can, ${ }^{\dagger}$ Erden Kilic, ${ }^{\S}$ Huseyin Ozkan, \\ Ilghar Orujalipoor," Semra Ide," Ayse B. Tekinay, ${ }^{, \dagger}$ and Mustafa O. Guler ${ }^{*, \dagger}$ \\ ${ }^{\dagger}$ Institute of Materials Science and Nanotechnology, National Nanotechnology Research Center (UNAM), Bilkent University, Ankara
06800, Turkey
${ }^{\ddagger}$ Department of Orthopaedics and Traumatology, Gulhane Military Medical Academy, Ankara 06010, Turkey
${ }^{\S}$ Yuzuncuyil Hospital, Ankara 06530, Turkey
${ }^{\|}$Department of Nanotechnology and Nanomedicine and Department of Physics Engineering, Hacettepe University, Ankara 06800,
Turkey
}

Supporting Information

ABSTRACT: Glycosaminoglycans (GAGs) and glycoproteins are vital components of the extracellular matrix, directing cell proliferation, differentiation, and migration and tissue homeostasis. Here, we demonstrate supramolecular GAG-like glycopeptide nanofibers mimicking bioactive functions of natural hyaluronic acid molecules. Self-assembly of the glycopeptide amphiphile molecules enable organization of glucose residues in close proximity on a nanoscale structure forming a supramolecular GAG-like system. Our in vitro culture results indicated that the glycopeptide nanofibers are

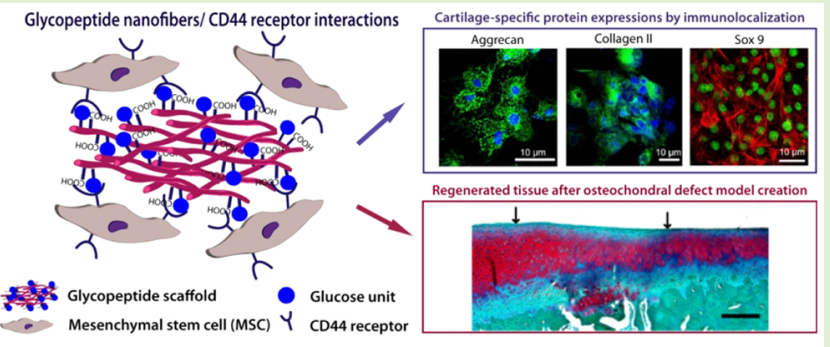
recognized through CD44 receptors, and promote chondrogenic differentiation of mesenchymal stem cells. We analyzed the bioactivity of GAG-like glycopeptide nanofibers in chondrogenic differentiation and injury models because hyaluronic acid is a major component of articular cartilage. Capacity of glycopeptide nanofibers on in vivo cartilage regeneration was demonstrated in microfracture treated osteochondral defect healing. The glycopeptide nanofibers act as a cell-instructive synthetic counterpart of hyaluronic acid, and they can be used in stem cell-based cartilage regeneration therapies.

\section{INTRODUCTION}

Adult cartilage tissue lacks the innate repair responses required for its complete regeneration. Many processes involved in cartilage development are lost or partially activated in the mature tissue, which prevents the recovery of cartilage injuries and allows their progressive degeneration into osteoarthritis. ${ }^{1,2}$ Therefore, repair of cartilage defects is a topic of great interest for the field of regenerative medicine. Complete and functional repair of any tissue requires an adequate supply of progenitor cells to produce a specialized extracellular matrix by recapitulating the tissue development process, which is characterized by cellular self-organization and lineage commitment through molecular specification. ${ }^{3}$ Consequently, the modulation of the cellular behavior through bioactive signals is an effective means of enhancing tissue repair. Mesenchymal stem cells (MSCs) are suitable progenitors for the repair of cartilage, and can follow the natural course of early cartilage development when stimulated by critical signals found in the cell microenvironment. Bone marrow-stimulating techniques utilize the patient's own population of MSCs to facilitate the repair of cartilage tissue. ${ }^{4}$ However, the outcome is generally fibrous cartilage replacement in the defect site that severely compromises tissue function, ${ }^{5}$ which indicates the deficiency in bioactive signals to sustain proper ECM production. Therefore, a suitable set of bioactive signals is necessary for the production of a healthy extracellular matrix and the maintenance of the chondrogenic phenotype by differentiating MSCs. Due to its inherent capacity for cellular recognition, regulatory role in developmental condensation, and high abundance in the native cartilage extracellular matrix, hyaluronic acid (HA) is used as an inductive microenvironment for the enhancement of cartilage repair. $^{6-8}$ Mesenchymal condensation, an essential stage of chondrogenesis, is tightly regulated by the distribution and organization of HA molecules, and specific HA-cell interaction coincides with the onset of condensation. ${ }^{9}$ HA mediates the crossbridging of cells into condensate units by binding to its transmembrane cell surface receptor, CD44. Downstream effectors of this receptor are responsible for initiating chondrogenesis, ${ }^{10}$ and the perturbation of HA-CD44 interactions may halt or delay the chondrogenic differentiation of MSCs. ${ }^{6} \mathrm{HA}-$ cell interactions therefore play a major role in

Received: December 11, 2015

Revised: December 23, 2015

Published: December 30, 2015 
chondrogenesis and the subsequent maintenance of the chondrogenic phenotype.

Although HA has been shown to enhance chondrogenesis under both in vitro and in vivo conditions, it nonetheless bears the limitations and drawbacks of naturally derived materials. In addition to the high costs and potential batch-to-batch variances associated with their extraction, polysaccharides derived from animals may also cause chronic immunogenic responses when introduced to the human body. ${ }^{1-13}$ Furthermore, cross-linking reagents used to produce HA derivatives are also toxic, and while polymer-based synthetic HA hydrogels have been developed, toxicity and degradability issues associated with these materials due to the applied crosslinking techniques prevent their application in in vivo systems. ${ }^{14}$ Although protein-glycosaminoglycan conjugates offer improved scaffold properties in cartilage, bioactivity pertaining to the core protein is often lost. ${ }^{15}$ There are attempts to develop supramolecular glycopeptide nanostructures and use them as biocompatible materials in biological applications. So far, various glycosyl units were incorporated into Fmoc- or naphthalene-conjugated amphiphilic di- or tripeptides to obtain self-supportive glycopeptide gels through noncovalent forces. However, attachment of saccharide unit to peptide backbone was achieved by using different chemical approaches, all of which lacked the glycosylation bonds found in natural systems. ${ }^{16-19}$ Previously, glycopolypeptides, as polymeric analogues of natural glycoproteins, were synthesized, and the hydrogels were also used as synthetic scaffolds for cartilage tissue engineering. ${ }^{20,21}$

Peptide amphiphile (PA) molecules combining the structural properties of amphiphilic surfactants with the bioactive peptides have created considerable interest not only because of their unique nanostructured features but also because they are biocompatible and biodegradable. ${ }^{22,23}$ Their self-assembly process is dictated by various noncovalent interactions resulting in the formation of high aspect ratio nanofibers under controlled conditions. ${ }^{24,25}$ Taken together, they are attractive candidates for diverse biomedical applications including drug delivery, wound healing, tissue engineering and regenerative medicine. ${ }^{26-30}$

In this work, we show a self-assembled glycopeptide nanofiber system, which has been devised to serve as an analogue of HA. The coassembly of a Ser-linked $\beta$-D-glucose containing amphiphilic glycopeptide and a carboxylic acidbearing PA results in formation of a synthetic HA emulating system. Self-assembly of the glycopeptide amphiphile molecules enable organization of multiple glucose residues in close proximity on a nanoscale supramoleculer polymeric system. The self-assembled glycopeptide nanofibers were observed to interact with MSCs through CD44 receptors and induce chondrogenic differentiation in a manner similar to native HA. In addition, an in vivo microfracture-treated osteochondral defect model was used to evaluate the effect of glycopeptide nanofiber hydrogels in promoting formation of hyaline-like cartilage as opposed to fibrous cartilage.

\section{EXPERIMENTAL SECTION}

Materials. 9-Fluorenylmethoxycarbonyl (Fmoc) and tert-butoxycarbonyl (Boc) protected amino acids except glyco amino acid, [4- $[\alpha-$ $\left(2^{\prime}, 4^{\prime}\right.$-dimethoxyphenyl) Fmoc-aminomethyl]phenoxy]acetamidonorleucyl-MBHA resin (Rink amide MBHA resin), Wang resin, and 2-(1H-benzotriazol-1-yl)-1,1,3,3 tetramethyluronium hexafluorophosphate (HBTU) were purchased from NovaBiochem. Fmoc-
$\operatorname{Ser}[\beta$-Glc $(\mathrm{OAc}) 4]-\mathrm{OH}$ was purchased from AAPPTec. Lauric acid and $N, N$ - diisopropylethylamine (DIEA) were purchased from Merck. Other chemicals were purchased from Alfa Aesar or Sigma-Aldrich and used without any purification. Water used during the experiments was deionized by Millipore Milli-Q with a resistance of $18 \mathrm{M} \Omega \cdot \mathrm{cm}$.

Synthesis and Characterization of Glycopeptide and Peptide Amphiphiles. Fmoc, Boc, protected amino acids, Wang resin, and MBHA Rink Amide resin were purchased from NovaBiochem. HBTU and Fmoc-Ser[beta-Glc(OAc) $\left.)_{4}\right]-\mathrm{OH}$ were purchased from $A B C R$ and Aapptec, respectively. The other chemicals were purchased from Alfa Aesar and Sigma-Aldrich and used as provided.

Protected glycopeptide was conjugated to the MBHA Rink Amide resin. All amino acid couplings were performed with 2 equiv of Fmocprotected amino acid, 1.95 equiv of HBTU, and 3 equiv of DIEA in DMF for $3 \mathrm{~h}$. Fmoc deprotections were performed with $20 \%$ piperidine/dimethylformamide (DMF) solution for $20 \mathrm{~min}$. The cleavage of the peptides from the resin and deprotection of acid labile protected amino acids were carried out with a mixture of trifluoroacetic acid (TFA):triisoproplysilane (TIS):water in the ratio of 95:2.5:2.5 for 2.5 h. Excess TFA was removed by rotary evaporation. The remaining residue was triturated with ice-cold diethyl ether, and the resulting white pellet was freeze-dried. The protected glycopeptide was identified and analyzed by reverse phase HPLC on an Agilent 6530 accurate-Mass Q-TOF LC/MS equipped with an Agilent 1200 HPLC. A Phenomenex Luna $3 \mu$ C8 100A $(50 \times 3.00 \mathrm{~mm})$ column as stationary phase and water/acetonitrile gradient with $0.1 \%$ volume of formic acid as mobile phase were used to identify protected amphiphilic glycopeptide. For the cleavage of acetyl groups, $210 \mathrm{mg}$ of protected glycopeptide ( 1 equiv) was dissolved in $105 \mathrm{~mL}$ of anhydrous methanol. Two molar $\mathrm{NaOMe}$ (4.4 equiv) was dissolved in methanol and poured into the solution. After adjusting the $\mathrm{pH}$ to $8-$ 8.5 , the reaction was carried out at room temperature for $2-3 \mathrm{~h}$. To stop the reaction, the solution was neutralized with a few drops of acetic acid, and the resulting compound was concentrated by rotary evaporation. After water addition, it was frozen at $-80{ }^{\circ} \mathrm{C}$ and freezedried. The deprotected glycopeptide was identified and analyzed by reverse phase HPLC on an Agilent 6530 accurate-Mass Q-TOF LC/ MS equipped with an Agilent 1200 HPLC. A Phenomenex Luna $3 \mu$ C8 $100 \mathrm{~A}(50 \times 3.00 \mathrm{~mm})$ column as stationary phase and water/ acetonitrile gradient with $0.1 \%$ volume of formic acid as mobile phase were used to identify the peptide amphiphile. The glycopeptide was purified on Agilent 1200 HPLC system by using a Zorbax prepHT $300 \mathrm{CB}-\mathrm{C} 8$ column with a water-acetonitrile $(0.1 \%$ TFA) gradient. KPA was synthesized and purified as indicated above. For E-PA synthesis, $1.1 \mathrm{mmol} / \mathrm{g}$ loaded Wang resin was preloaded with Fmoc$\mathrm{Glu}(\mathrm{OtBu})-\mathrm{OH}$, and the resultant resin had $0.72 \mathrm{mmol} / \mathrm{g}$ loading. The rest of the synthesis procedure was identical for E-PA. Due to its acidic character, an Agilent 6530 accurate-Mass Q-TOF LC/MS was operated by eluting it from an Agilent Zorbax Extend-C18 $(50 \times 2.1$ $\mathrm{mm})$ column with a water/acetonitrile mixture $\left(0.1 \% \mathrm{NH}_{4} \mathrm{OH}\right)$ for the elucidation of the molecule. The purification was performed on a Zorbax Extend C18 prep-HT with a water/acetonitrile $(0.1 \%$ $\left.\mathrm{NH}_{4} \mathrm{OH}\right)$ gradient.

Gel Preparation. Oppositely charged glycopeptide or peptide amphiphiles $(10 \mathrm{mM})$ were mixed at a specified ratio $(1: 1$ or $2: 1)$ to obtain neutral or negatively charged supramolecular gels. While Glc$\mathrm{PA}$ and K-PA were positively charged at physiological $\mathrm{pH}, \mathrm{E}-\mathrm{PA}$ exhibited negative charge due to the acidic character of the molecule. When they are mixed, oppositely charged peptides form threedimensional networks as a result of interactions such as hydrogen bonding, van der Waals, and electrostatic forces.

Circular Dichroism (CD). Secondary structures of PA molecules and coassembled PA and HA nanofibers were investigated with CD. A Jasco J-815 CD spectrophotometer was used for CD analysis. All samples were measured at physiological $\mathrm{pH}$ at $0.25 \mathrm{mM}$ concentration. PA molecules prepared at physiological $\mathrm{pH}$ were sonicated one by one prior to mixing. For each measurement, $300 \mu \mathrm{L}$ of the sample was transferred into a $1 \mathrm{~mm}$ quartz cuvette, which was inverted gently for mixing without damaging the assembled structures. Spectra were 
obtained at room temperature from 300 to $190 \mathrm{~nm}$ with a data interval of $1 \mathrm{~nm}$ and a scanning speed of $100 \mathrm{~nm} / \mathrm{min}$. The results were expressed as mean residue ellipticity and converted to the unit of deg. $\mathrm{cm}^{2} \cdot \mathrm{dmol}^{-1}$.

Determination of Zeta Potentials of Nanofiber Systems. Samples were prepared by dissolving each component in water at a concentration of $0.250 \mathrm{mM}$ and measuring either individual peptide solutions or Glc-PA/E-PA $(1: 1 \mathrm{v} / \mathrm{v} \%)$ and E-PA/K-PA (1:2 v/v\%) mixtures. Zeta potentials of the peptides were measured using a Malvern Nanosizer/Zetasizer nano-ZS ZEN 3600 (Malvern Instruments, USA) instrument. Measurements were performed in glass cuvettes and repeated at least three times.

Transmission Electron Microscopy (TEM) Imaging. TEM images were obtained with a FEI Tecnai G2 F30 TEM at $200 \mathrm{kV}$. A high-angle annular dark field (HAADF) detector was used for images taken in STEM mode. Ten millimolar Glc-PA/E-PA, K-PA/E-PA and HA/K-PA nanofiber systems were first diluted 100-200 times and then dropped on a 200-mesh copper TEM grid. Samples were left at room temperature for $5 \mathrm{~min}$, stained by $2 \mathrm{wt} \%$ uranyl-acetate staining for another 3-4 min, and air-dried prior to TEM imaging.

Scanning Electron Microscopy (SEM) Imaging. For SEM imaging, samples were prepared on cleaned silicon wafer by mixing 10 $\mathrm{mM}$ Glc-PA and E-PA at 1:1 ratio, $10 \mathrm{mM} \mathrm{K}-\mathrm{PA}$ and E-PA at 2:1 ratio, and $10 \mathrm{mM}$ HA and K-PA at 1:1 ratio. Samples were incubated at room temperature for $20 \mathrm{~min}$ for gelation. To preserve their initial network structures, the dehydration of samples was performed by immersing silicon wafers into gradually increasing concentrations of ethanol solutions. After solvent exchange, samples were dried using a Tourismis Autosamdri-815B critical point drier. SEM imaging was performed with a FEI Quanta 200 FEG, using the GSED detector at ESEM mode with 3-10 keV beam energy. Samples were coated with 5 $\mathrm{nm}$ of $\mathrm{Au}-\mathrm{Pd}$ prior to imaging.

Small Angle X-ray Scattering (SAXS). SAXS measurements were performed with a Kratky compact HECUS (Hecus X-ray systems, Graz, Austria) system for $1 \mathrm{mM}$ Glc-PA/E-PA (1:1) and K-PA/E-PA $(2: 1)$ nanofiber systems. Solutions were directly loaded to quartz capillary cells. The SAXS apparatus consisted of a linear collimation system, a linear-position sensitive detector (PSD), an X-ray tube $\mathrm{Cu}$ target $(\lambda=1.54 \AA)$, and a generator operating at a power of $2 \mathrm{~kW}(50$ $\mathrm{kV}$ and $40 \mathrm{~mA}$ ). Distances between 1024 channels (in PSD) and the sample-detector were $54 \mu \mathrm{m}$ and $28.1 \mathrm{~cm}$, respectively. Scattering curves were monitored in the $q$ ranges of $0.002-0.55 \AA^{-1}$. SAXS measurements were carried out at room temperature $\left(23^{\circ} \mathrm{C}\right)$, and the data collection time was $900 \mathrm{~s}$ for each sample. The results were deconvoluted with the slit width and slit length profiles of the primary beam by using the related smearing process of the used HECUS SWAXS system.

Investigation of Viscoelastic Behavior of Nanofiber Systems by Oscillatory Rheology. Viscoelastic properties of the PA systems were analyzed with an Anton Paar Physica RM301 Rheometer operating with a $25 \mathrm{~mm}$ parallel plate configuration. Ten millimolar KPA and E-PA were mixed in 2:1 ratio to form a neutral hydrogel system, while equal concentrations of Glc-PA and E-PA were mixed in 1:1 ratio to form negatively charged (overall -1 charge) gels. Rheological analyses were also performed in biological media. While Glc-PA and K-PA were dissolved in $0.25 \mathrm{M}$ sucrose, E-PA was dissolved in DMEM. The ratios used for experiments in biological media were identical to these in water. A gap distance of $0.5 \mathrm{~mm}$ was used with angular frequency of $10 \mathrm{rad} / \mathrm{s}$, and shear strain of $0.1 \%$. All time-sweep experiments were carried out at room temperature. Measurements were performed with three replicates.

Mouse Mesenchymal Stem Cell (mMSC) Culturing and Preparation of Nanofibrous Networks for in Vitro Culture. mMSCs were expanded to passage 3 in maintenance medium consisting of DMEM with 10\% (v/v) FBS (Invitrogen), 1\% (v/v) GlutaMAX (Invitrogen) and 1\% penicilin-streptomycin (Invitrogen). All experiments were conducted with cells within passage 3-8. Cells were maintained in humidified incubators at $5 \% \mathrm{CO}_{2}$ at $37^{\circ} \mathrm{C}$. Cells were passaged when they reached $80 \%$ confluency through detachment by Trypsin-EDTA (0.025\%) (Invitrogen) and reseeding at 3000 cells $/ \mathrm{cm}^{2}$. For in vitro analysis, mMSCs were cultured on tissue culture plate or surfaces coated with Glc-PA/E-PA, K-PA/E-PA, or HA/K-PA. Coating was performed with $1 \mathrm{mM}$ PA solutions or HA solution prepared from sodium salt (Sigma, Cat no: 42686). Coated plates were left under laminar flow hood to dry for $16 \mathrm{~h}$ and sterilized under UV irradiation for $30 \mathrm{~min}$ prior to cell seeding.

Viability, Proliferation, and SEM Imaging. Cellular viability was assessed by colorimetric MTT assay (Sigma, Cat no:.TOX-1). Cells were seeded at a density of 250 cells $/ \mathrm{cm}^{2}$, and cultured for $24 \mathrm{~h}, 48$ and $72 \mathrm{~h}$ at parallel plates. At the time of the assay, cells were incubated with (3-(4,5-dimethylthiazol-2-yl)-2,5 diphenyltetrazolium bromide (MTT) reagent (Sigma-Aldrich) for $3 \mathrm{~h}$ at standard cell culture conditions. Viable cells form a purple formazan product through the reduction of the MTT reagent. Cell viability was quantified by the spectrophotometric measurement of solubilized formazan products at $590 \mathrm{~nm}$. Viability was normalized against the tissue culture plate. Proliferating cells were detected by Click-iT EdU assay (Molecular Probes) at days 1 and 2. mMSCs were seeded on nanofibers or culture plate at a concentration of $250 \mathrm{cells} / \mathrm{cm}^{2}$ in maintenance medium. After $6 \mathrm{~h}$, maintenance medium was exchanged with maintenance medium supplemented with $10 \mathrm{mM}$ EdU. At the time of the assay, cells were fixed with $4 \%$ paraformaldehyde/PBS and permeabilized with 5\% Triton-X. To detect incorporated EdU in proliferating cell DNA, cells were treated with Alexa Fluor-488conjugated azide. Cells stained with Alexa Fluor- 488 were imaged by a fluorescence microscope and quantified with ImageJ software. Proliferation rate of cells on Glc-PA/E-PA, K-PA/E-PA, and HA/K$\mathrm{PA}$ was normalized to cells on a tissue culture plate.

For SEM imaging, mMSCs were cultured for 7 days in maintenance medium, culture plate wells were washed with PBS, and the attached cells were fixed with $2 \%$ gluteraldehyde/PBS for $2 \mathrm{~h}$. Following three washing steps with PBS, samples were dehydrated in a series of ethanol solutions starting with $20 \%$ ethanol and proceeding to absolute ethanol for $10 \mathrm{~min}$ at each step. Samples were dried with a Tourismis Autosamdri-815B critical point drier, coated with $6 \mathrm{~nm} \mathrm{Au} /$ Pd and imaged with a FEI Quanta 200 FEG SEM.

Glycosaminoglycan Quantification. Quantification of sGAGs was performed by a biochemical dimethylmethylene blue assay. ${ }^{31}$ Cell cultures were digested in papain digestion buffer $(100 \mathrm{mM}$ sodium phosphate buffer $/ 10 \mathrm{mM} \mathrm{Na}{ }_{2}$ EDTA $/ 10 \mathrm{mM}$ L-cysteine $/ 0.125 \mathrm{mg} / \mathrm{mL}$ papain) overnight at $65{ }^{\circ} \mathrm{C}$ prior to analysis. Total DNA per well was measured with a Qubit dsDNA quantitation kit (Invitrogen) according to manufacturer's instructions. Total dsDNA amount was used to normalize the sulfated glycosaminoglycan content. Diluted chondroitin sulfate standards (from 0 to $35 \mu \mathrm{g} \mathrm{mL}^{-1}$ ) were used to generate standard curves for the DMMB assay. A total of $100 \mu \mathrm{L}$ of DMMB solution (16 mg L $\mathrm{m}^{-1} 1$,9-dimethylmethylene blue, $40 \mathrm{mM}$ glycine, 40 $\mathrm{mM} \mathrm{NaCl}, 9.5 \mathrm{mM} \mathrm{HCl}, \mathrm{pH} 3.0)$ was added to $40 \mu \mathrm{L}$ of papaindigested solutions and standard samples, and optical densities (ODs) of the solutions were measured using a $595 \mathrm{~nm}$ filter on a microplate reader. The absorbance of the cell-free control groups was subtracted from the absorbance values of the experimental groups.

Gene Expression Analysis. Gene expression profiles for analyzing chondrogenic differentiation were assessed by quantitative real time PCR (qRT-PCR). Before qRT-PCR experiments, RNA from each sample was extracted with TRIzol reagent (Invitrogen) according to manufacturer's instructions. The yield and purity of extracted RNA were assessed with Nanodrop 2000 spectrophotometer (Thermo Scientific). cDNA synthesis from RNA and qRT-PCR were performed using SuperScript III Platinum SYBR Green One-Step qRT-PCR Kit according to manufacturer's instructions. Reaction conditions were briefly as follows: $55^{\circ} \mathrm{C}$ for $5 \mathrm{~min}, 95^{\circ} \mathrm{C}$ for $5 \mathrm{~min}, 40$ cycles of $95{ }^{\circ} \mathrm{C}$ for $15 \mathrm{~s}, 60^{\circ} \mathrm{C}$ for $30 \mathrm{~s}$, and $40^{\circ} \mathrm{C}$ for $1 \mathrm{~min}$, followed by a melting curve to confirm product specificity. The reaction efficiencies for each primer set were evaluated with a standard curve using 5-fold serial dilutions of total RNA. Each run was internally normalized to GAPDH, and each group was normalized to the expression levels of mesenchymal stem cells cultured in maintenance medium. A comparative $\mathrm{Ct}$ method with efficiency correction was used to analyze the results. An expression ratio of greater than 1 indicates 
a

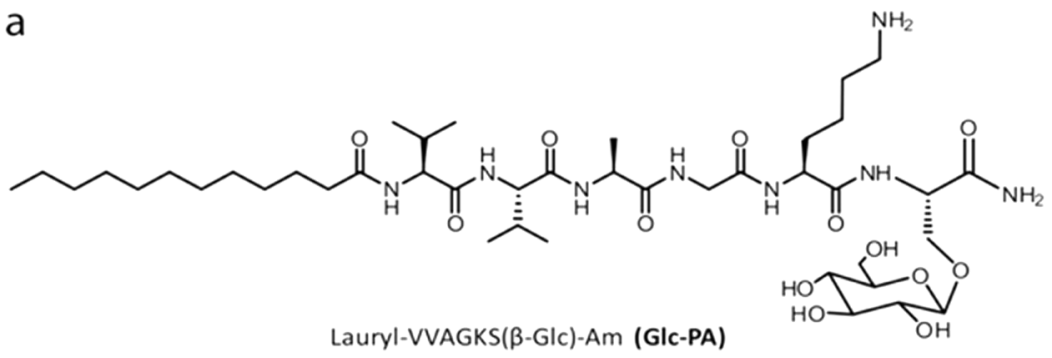

Lauryl-VVAGKS(B-GIC)-Am (GIc-PA)<smiles>CCCCCCCCCCCC(=O)NC(C(=O)N[C@H](C(=O)NC(C)C(=O)NCC(=O)NC(CCC(=O)O)C(=O)O)C(C)C)C(C)C</smiles><smiles>CCCCCCCCCCCC(=O)NC(C(=O)N[C@H](C(=O)NC(C)C(=O)NCC(=O)NC(CCCCN)C(N)=O)C(C)C)C(C)C</smiles>

Lauryl-VVAGK-Am(K-PA)
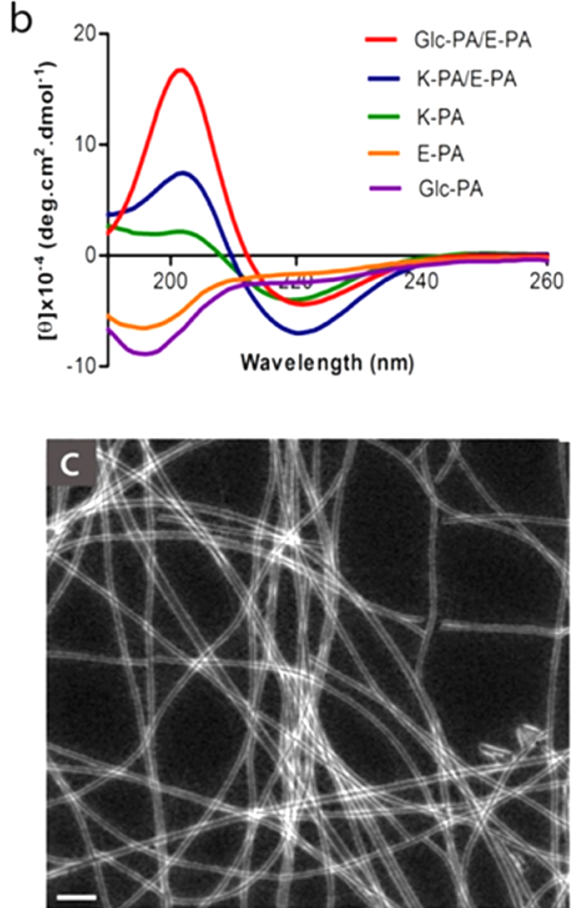

Figure 1. Design of peptide amphiphile molecules (PA). (a) Chemical structures of molecules. (b) Circular dichroism spectra of peptide amphiphile solutions and nanofiber systems. (c) STEM image of Glc-PA/E-PA nanofibers; scale bar $=100 \mathrm{~nm}$.

upregulation, while a ratio less than 1 corresponds to the downregulation of the gene of interest.

Primers Used for qRT-PCR Expression Analysis. Col I; F:5' TGACTGGAAGAGCGGAG AGT-3', R:5'-GTTCGGGCTGATGTACCAGT-3', Col II; F:5'-ACTTGCGTCTACCCCAACC-3', R:5'GCCATAGCTGAAGTGGAAGC-3', Aggrecan; F: 5'-GGTCACTGTTACCGCCACTT-3', R: 5'-CCCCTTCGATAGTCCTGTCA3', Sox-9; F: 5'-AGGAAGCTGGCAGACCAGTA-3', R: 5'CGTTCTTCACCGACTTCCTC-3', Hyal-1; F: 5'-ATGCCCTTTACCCCAGTATT-3', R: 5'TGGGGGTCTCTGGAACTAT-3'.

Immunostaining and Imaging. mMSCs were fixed in $4 \%$ paraformaldehyde/PBS for $10 \mathrm{~min}$ and permeabilized in $0.1 \%$ Triton X-100 for 15 min. For blocking, samples were incubated with $10 \%(\mathrm{w} /$ v) bovine serum albumin/PBS for $30 \mathrm{~min}$ and treated with collagen II primary antibody (Abcam) at 1:200 dilution, Sox-9 primary antibody (Thermoscientific) at 1:300 dilution or aggrecan antibody at 1:200 dilution (Abcam) overnight at $4{ }^{\circ} \mathrm{C}$. Cells were then washed with PBS and incubated for $1 \mathrm{~h}$ at room temperature with Goat Anti-Rabbit IgG H\&L (Alexa Fluor 488). All samples were counterstained with $1 \mu \mathrm{M}$ TO-PRO-3 (Invitrogen) in PBS for $15 \mathrm{~min}$ at room temperature and mounted with Prolong Gold Antifade Reagent (Invitrogen). Negative controls were obtained by omitting the primary antibody and incubating with $1 \%$ normal goat serum/PBS. Samples were imaged by confocal microscopy (Zeiss LSM510).

In Vitro 3D Cell Viability Analysis and Morphology Characterization. Collagen 1 (Life Technologies) gel was used as control in 3D cell culture studies. Negatively charged PA molecules were dissolved in cell media (DMEM $+10 \% \mathrm{FBS})$, and positively charge PA molecules were dissolved in $0.25 \mathrm{M}$ sucrose solution. mMSC cell pellets were suspended into E-PA solution (with culture media) and mixed inside wells alongside Glc-PA or K-PA to produce 3D scaffolds. Three milliliters of cell medium was added to the wells, and the gels were incubated for $30 \mathrm{~min}$ at $37{ }^{\circ} \mathrm{C}$ prior to cell seeding. The final concentrations of PA molecules were as follows: $10 \mathrm{mM}$ GlcPA, $5 \mathrm{mM}$ E-PA, and $10 \mathrm{mM}$ K-PA. The final cell amount was $5 \times 10^{5}$ cells in each gel. Viabilities of mMSCs seeded in PA gels were analyzed by using Alamar Blue Assay (Invitrogen) on days 3, 7, 14, and 21 . Viable cell amounts were quantified by spectrophotometry. Live/Dead Assay was also performed for $3 \mathrm{D}$ mMSC cultures in bioactive and control hydrogels. For SEM imaging, 3D PA gels were washed with PBS and fixed with $2 \%$ gluteraldehyde/PBS for $2 \mathrm{~h}$. Following three washing steps with PBS, samples were dehydrated in graded ethanol solutions starting with $20 \%$ ethanol and proceeding to absolute ethanol for $10 \mathrm{~min}$ at each step. Samples were dried with a Tourismis Autosamdri-815B critical point drier, coated with $6 \mathrm{~nm} \mathrm{Au} / \mathrm{Pd}$ and imaged with FEI Quanta 200 FEG SEM.

In Vivo Osteochondral Defect Model and Treated with Microfracture Treatment. Twelve white male New Zealand rabbits (mean weight $2500 \pm 400 \mathrm{~g}$, age 12 weeks) were used for in vivo experiments. All procedures on animals were approved by the Gulhane Military Medical Academy (GATA) Animal Ethics Committee. Animals were anesthetized by intramuscular injection of $30-40 \mathrm{mg} /$ $\mathrm{kg}$ ketamine and 5-7 mg/kg xylazine prior to surgery. The region of operation was shaved and aseptically prepared for operation. A lateral parapatellar longitudinal incision was made to expose the knee joint, the synovial capsule was incised, and the trochlear groove was exposed after the medial luxation of the patella. When the knee was maximally flexed, a defect $(1.5 \mathrm{~mm}$ in diameter, and $1.5 \mathrm{~mm}$ in depth and diameter) was created in the center of the groove using a drill. All debris, including articular cartilage, was removed with a micro curet. Microfracture treatment was performed by creating three holes within each defect with a sterile needle to mobilize bone marrow blood. After observing blood flow from holes, defects were filled with physiological saline (saline-treated), $100 \mu \mathrm{L}$ of Glc-PA/E-PA or a clinically approved formulation of HA (Hyalgan). The wound was closed by suturing (Vicryl 4-0 absorbable suture) of the knee joint capsule and the overlaying skin layer-by-layer. IM antibiotics were given to each rabbit for 3 days following the operation. All animals were carefully evaluated during the first $24 \mathrm{~h}$ after operation. No animals were observed to be infected throughout the experimental period. Rabbits were provided with individual cages and observed for 12 weeks prior to sacrifice. The rabbits were euthanized at 12 weeks with overdose sodium pentobarbital after sedation. Only one defect was created for each trochlea, and two different treatments were tested for each rabbit. A total of eight trochleae were used for each treatment; however, two were later excluded due to improper handling.

Histological and Immunohistochemical Stainings of Tissue Sections. Samples were fixed in $4 \%$ paraformaldeyde for $48 \mathrm{~h}$ at $4{ }^{\circ} \mathrm{C}$ 
a

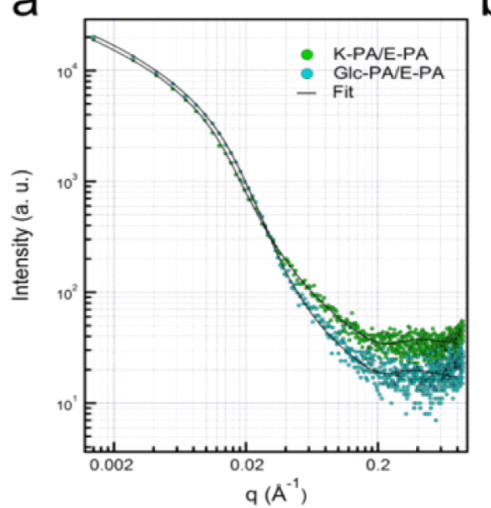

C

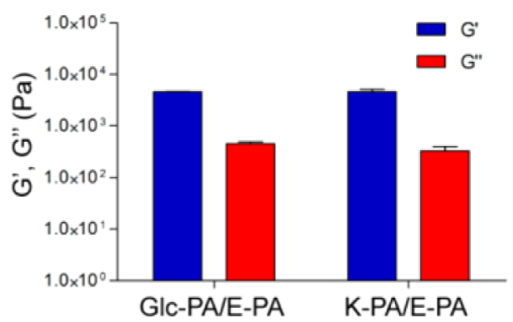

b

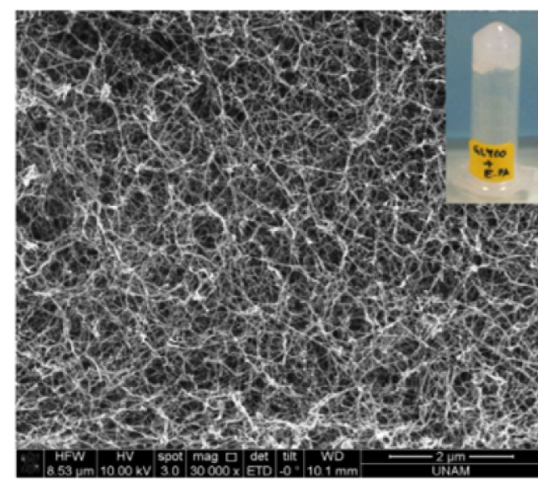

d

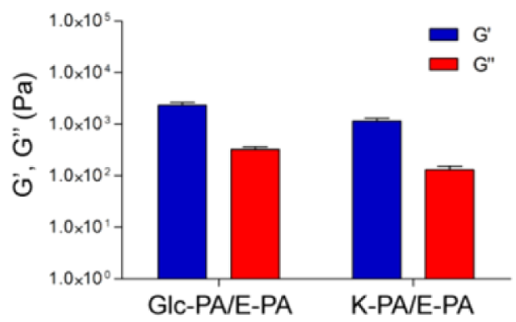

Figure 2. Structural, morphological, and mechanical properties of the nanofiber networks. (a) SAXS profile and fit curve for the scattering data of Glc-PA/E-PA and K-PA/E-PA nanofibers. (b) SEM micrograph of Glc-PA/E-PA nanofibers, scale bar $=2 \mu$ m, inset photo shows self-supportive Glc-PA/E-PA (1\% w/v) glycopeptide gel. (c,d) Equilibrium storage and loss moduli of Glc-PA/E-PA and K-PA/E-PA nanofibers in water at pH 7 (c) and in $0.25 \mathrm{M}$ sucrose/DMEM medium (d).

and decalcified in $5 \%$ formic acid. The $5 \%$ formic acid solution was changed every 2-3 days. The completion of decalcification was periodically tested with ammonium oxalate test. Decalcified samples were dehydrated in a graded series of ethanol and cleared in two changes of xylene. Samples were embedded in paraffin blocks and sectioned at $5 \mu \mathrm{m}$ thickness by microtome. For histological and immunohistochemical evaluations, sections were deparaffinized and rehydrated through a series of graded alcohol solutions. In order to track defect progression and comparison with healthy cartilage, consecutive sections at every 10 section from each experimental group were stained with hematoxylin and eosin (H\&E) (Figure S15). For GAG imaging, sections were stained with Safranin-O for 5 min and Fast Green for $5 \mathrm{~min}$ and for H\&E staining sections stained with Mayer's haematoxylin for $5 \mathrm{~min}$ and eosin for $45 \mathrm{~s}$. Then sections dehydrated in graded ethanol solutions and cleared in xylene. Slides were mounted by Histomount mounting medium and imaged by light microscopy (Zeiss, Axio Scope). For immunohistochemical stainings, slides were treated with an antigen retriever (Sigma) to uncover epitopes for $15 \mathrm{~min}$ at $37^{\circ} \mathrm{C}$ after rehydration steps. After blocking for $2 \mathrm{~h}$ at room temperature, sections were incubated with primary collagen II antibody (Pierce Antibodies) at $4{ }^{\circ} \mathrm{C}$ overnight. Sections were then washed extensively with TBS $\mathrm{w} /$ Triton-X $(0.01 \% \mathrm{vol} / \mathrm{vol})$ and treated with Goat anti-Mouse IgG-HRP at a dilution of 1:500 for 1 $\mathrm{h}$ at room temperature to detect bound primary antibodies. Secondary antibody binding was visualized with diaminobenzidine (DAB), and nuclei were stained with hematoxylin (Sigma-Aldrich). Collagen II antibody was tested on positive control samples before staining of sections from experimental groups (Figure S16). Negative controls were obtained by omitting primary antibody and incubating with $1 \%$ normal goat serum/TBS. Slides were mounted with Histomount mounting medium.

Statistical Analysis. All data were presented as means \pm standard error of means (s.e.m). Either one-way ANOVA or two-way ANOVA with post tests Tukey's/Bonferroni was performed to test the significance of observed differences between the study groups. A value of $p<0.05$ was considered to be statistically significant, except where noted.

\section{RESULTS AND DISCUSSION}

Glc-PA (Figure 1a) was synthesized by conjugation of a Serlinked, acetyl-protected $\beta$-D-glucose glyco-amino acid to the solid support prior to the synthesis of the peptide molecule. The glucose residue is present at the $\mathrm{C}$-terminus of the peptide amphiphile molecule, while the hydrophobic character of the molecule was provided by conjugation of a fatty acid to the $\mathrm{N}$ terminus. Deacetylation of hydroxyl groups was performed after resin cleavage, in solution phase and in the presence of methanolic sodium methoxide, so as to prevent $O$-glycosidic bond cleavage during acid treatment. ${ }^{32}$ The other two PA molecules, E-PA and K-PA, were also synthesized by Fmoc solid-phase peptide synthesis method. The E-PA molecule bears a negative charge due to its glutamic acid residue and acts as a charge neutralizer for the self-assembly of the lysinebearing Glc-PA molecule, while K-PA was used as a nonbioactive replacement for Glc-PA in experimental controls. The chemical structures of all three molecules were confirmed with electrospray ionization mass spectrometry (Figure S1). The designed glycopeptide molecule was unique in term of its amphiphilic nature and capacity to self-assemble into nanofibers when oppositely charged PA molecule was introduced. Cohesive interactions between PA molecules, such as hydrogen bonding and van der Waals, hydrophobic, and electrostatic interactions, were the driving forces that promote the selfassembly process and enable the formation of three-dimensional nanofiber networks. ${ }^{23,33}$

The coassembled PA systems were studied by circular dichroism (CD) spectroscopy. The results revealed that coassembled systems were oriented in a $\beta$-sheet conformation, displaying a negative minimum at $220 \mathrm{~nm}$ and positive ellipticity at $202 \mathrm{~nm}$. In case of pure PAs, while Glc-PA and E-PA showed disordered conformation, K-PA exhibited $\beta$-sheet 
a

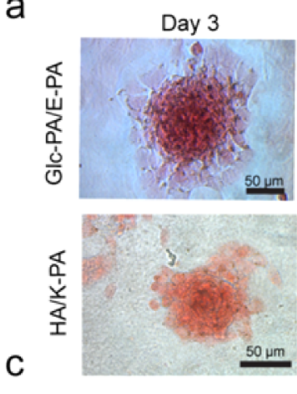

Collagen II

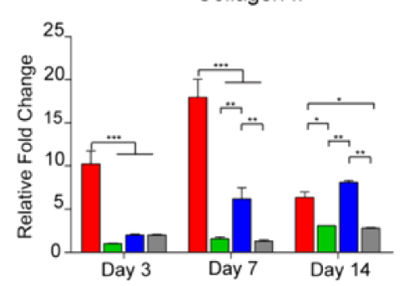

Day 7

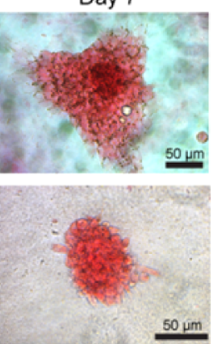

$50 \mathrm{\mu m}$
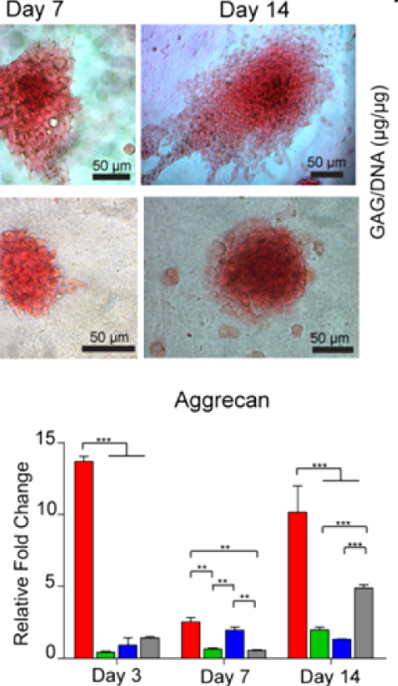

b

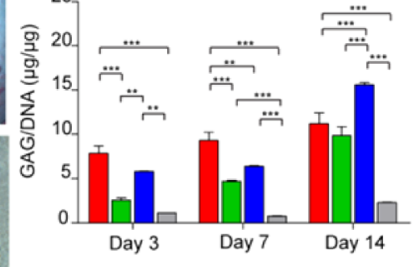

Sox 9

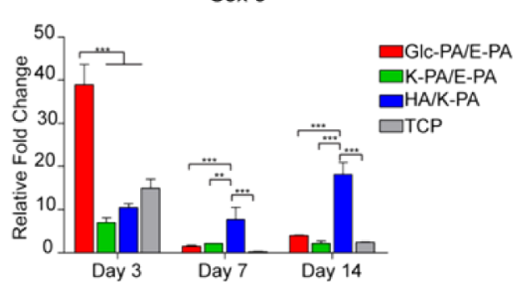

d
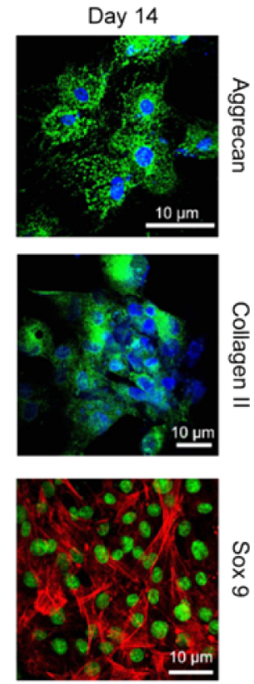

Figure 3. Glycopeptide nanofibrous networks enhance the chondrogenic differentiation of mMSCs. (a,b) Glycosaminoglycan (GAG) deposition was analyzed by Safranin-O staining and DMMB assay. Safranin-O stainings (a) and DMMB assay for quantification of GAGs (normalized to DNA amount) (b) demonstrate the elevated production of GAGs by mMSCs cultured on Glc-PA/E-PA and HA/K-PA on days 3, 7, and 14 in chondrogenic medium. (c) Gene expression analyses performed for Collagen II, Aggrecan and Sox 9 genes on days 3, 7, and 14 in chondrogenic medium. Expression ratio was normalized to GAPDH and calibrated to cultured cells on TCP in maintenance media. (d) Immunolocalization of Aggrecan, Collagen II and Sox 9 protein expressions of mMSCs on glycopeptide nanofibers on day 14 in chondrogenic medium. Error bars in b and c indicate SEM, $n=3, * *_{p}<0.05, * p<0.01$.

structure (Figure 1b). The coassembled PA nanofibers were observed under scanning transmission electron microscopy (STEM) and diluted gels contained high-aspect-ratio nanofibers with diameters in the order of 8-10 nm and lengths reaching several micrometers (Figures 1c and S2a). Small-angle $\mathrm{X}$-ray scattering (SAXS) analysis was also performed to obtain further information about the structural characteristics of PA nanofiber systems in the aqueous environments. The $1 \mathrm{mM}$ of Glc-PA/E-PA and K-PA/E-PA solutions were prepared at physiological $\mathrm{pH}$ and inserted into quartz capillaries for analysis. The SAXS data were analyzed using the $q$ region between 0.002 and $0.55 \AA^{-1}$ and flexible cylinder-polydisperse length model was found to be the best fitted model with fiber diameters of $9.2 \pm 0.1 \mathrm{~nm}$ and $8.9 \pm 0.1 \mathrm{~nm}$ for Glc-PA/E-PA and K-PA/E-PA, respectively (Figure $2 \mathrm{a}$ and Table S1). ${ }^{34,35}$ Pair distance distributions (PDDs) derived from SAXS analyses were also used to investigate the difference in the electron densities of Glc-PA/E-PA and K-PA/E-PA nanofibers (Figure S3). Although symmetrical shoulders were observed in the PDDs of both PA systems, sharper humps were obtained arising from the existence of glucose units in the case of GlcPA/E-PA, which are also evidence of more numbers of the scattered nanoscale aggregations and indirectly more electrondense regions.

Three-dimensional nanofiber networks of Glc-PA/E-PA and K-PA/E-PA gels were studied by using a scanning electron microscope. The structural organization of the nanofiber network was observed to resemble the native extracellular matrix that potentially provides an environment conducive for gathering chemical, physical, and biological cues (Figures $2 \mathrm{~b}$ and S2b). ${ }^{36}$ The mechanical properties of the nanofibrous hydrogels were examined by oscillatory rheology. A time sweep test was conducted by recording the storage $\left(G^{\prime}\right)$ and loss $\left(G^{\prime \prime}\right)$ moduli of the hydrogels for $1 \mathrm{~h}$ at constant shear strain and angular frequency to determine the viscoelastic behaviors of PA scaffolds. Oscillatory rheology measurements showed that storage moduli were greater than loss moduli for Glc-PA/EPA and K-PA/E-PA nanofibers prepared both in water at physiological $\mathrm{pH}$ and in sucrose/DMEM medium, suggesting that the nanofiber networks displayed elastic solid behavior (Figure 2c,d). These self-supporting hydrogels are suitable candidates for in vivo applications. ${ }^{26}$

We studied similarity of the glycopeptide nanofibers to HA by studying the recognition of the glycosaminoglycans through the CD44 receptor. CD44 receptor is responsible for cellular recognition and commitment to chondrogenic differentiation pathway. The Glc-PA/E-PA, HA/K-PA, K-PA/E-PA, and standard polystyrene culture plate groups were used for mMSC cultures. All three of the nanosystems displayed similar zeta potentials (Figure S4). HA/K-PA served as a biological positive control, while K-PA/E-PA was a nanofibrous gel control that bears the same functional units as Glc-PA/E-PA, except for the glycoamino acid residue. HA/K-PA was also characterized similar to the other two PA systems in terms of secondary structure and morphological properties (Figure S5). $\mathrm{CD}$ results revealed that HA alone exhibited a very weak signal, while the HA/K-PA mixture forms a $\beta$-sheet composition with a negative signal at around $220 \mathrm{~nm}$ and a positive signal below $200 \mathrm{~nm}$ (Figure S5c). The assembly of HA and K-PA was also observed by TEM and SEM imaging. HA/K-PA assemblies were found to form bundles with micrometer-scale lengths, and their nanoporous structure presumably allows the mimicking of the native ECM environment (Figure S5b,d).

In vitro viability and proliferation rates of mMSCs were evaluated to ensure that cell survival was not adversely affected on nanofibrous networks. Cellular viability was at comparable levels on days 1,2 , and 3 of culture, suggesting that the nanofibrous networks are biocompatible. The rate of proliferation of mMSCs was also comparable to control at 24 and $48 \mathrm{~h}$ of culture (Figure S6a,b). Cells were able to interact with the nanofibrous network and changed their morphology following their seeding onto the peptide nanofibers, as shown 

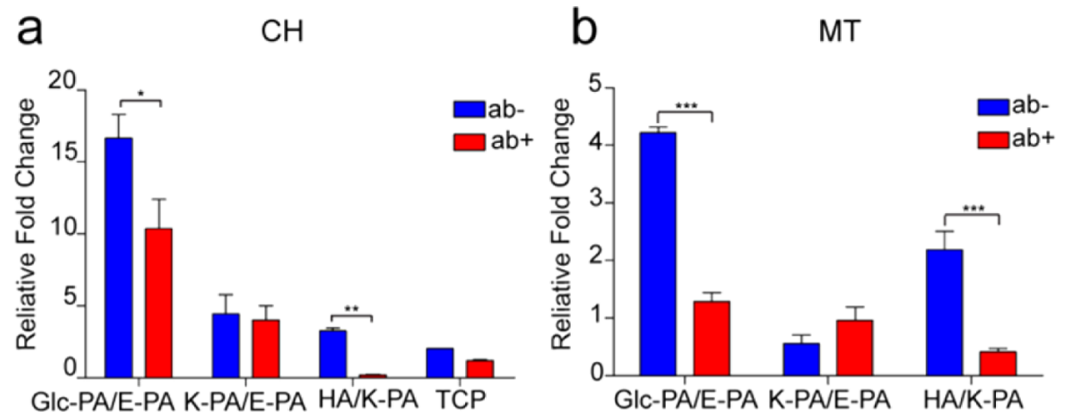

Figure 4. CD44 blocking downregulates Sox 9 expression of mMSCs on glycopeptide nanofibrous networks. (a,b) Sox 9 expression analysis was performed at day 1 using mMSCs cultured in two different media: chondrogenic differentiation (a) and maintenance medium (b) (anti-CD44 treatment indicated with $\mathrm{ab}+$; no treatment indicated with ab-). Expression ratio was normalized to GAPDH and calibrated to cultured cells on TCP in maintenance media. Error bars indicate SEM, $n=3, * * p<0.05, * p<0.01$.

by SEM images on day 7 (Figure S6c). Hyaluronidase-1 (Hyal1) expression was studied on day 2 of culture to determine whether cells recognize the Glc-PA/E-PA matrix through CD44 receptor-mediated interactions in a similar manner to the CD44-HA binding process. Hyal-1 is one of the main enzymes for the cleavage of HA and has been reported to be upregulated in response to the binding of CD44 to HA. ${ }^{37,38}$ The mRNA expression of Hyal-1 was enhanced in cells on Glc-PA/E-PA and $\mathrm{HA} / \mathrm{K}-\mathrm{PA}$, while no significant changes were observed for K-PA/E-PA. The blocking of CD44 by anti-CD44 antibody significantly downregulated the Hyal-1 expression of cells on Glc-PA/E-PA and HA/K-PA (Figure S6e).

To assess the influence of glycopeptide nanofibers on the chondrogenic differentiation of mMSCs, we cultured cells on nanofibrous networks over the course of 14 days and examined glycosaminoglycan production and the expression levels of genes and proteins involved in chondrogenesis during this time period. One of the key features of chondrogenic differentiation in mMSCs is the elevated level of cell-cell interactions. ${ }^{39}$ Longterm culture of mMSCs revealed that cells on nanofibrous networks undergo condensation to increase cell-cell contacts (Figure S7). This spontaneous aggregation of cells eliminates the necessity of micromass culture to induce in vitro chondrogenic differentiation. The mMSCs on Glc-PA/E-PA also exhibited a rounded morphology, which differs from their normal spindle/fibroblastoid shape, in both chondrogenic and growth media. This morphology was observed on days 3 through 14, suggesting that the cells preserve their phenotype over time (Figure S7).

Time-dependent tracking of sulfated glycosaminoglycan deposition and gene expression levels revealed that mMSCs on Glc-PA/E-PA committed to the chondrocytic lineage at a very early stage. On day 3 , the mMSCs on Glc-PA/E-PA were found to deposit significantly higher amounts of glycosaminoglycans than those cultured on HA/K-PA, K-PA/E-PA, and the culture plate in both chondrogenic and growth media (Figures $3 \mathrm{~b}$ and $\mathrm{S} 8 \mathrm{~b}$ ). Expression profile of chondrogenesis markers showed that mMSCs on Glc-PA/E-PA displayed significantly higher fold changes (Collagen II $=\sim 10$, Aggrecan $=\sim 14$, Sox $=$ $\sim 38$ ) in chondrogenesis-associated genes following 3 days of culture in chondrogenic medium, which is in accordance with the GAG deposition results. A similar result was observed, although to a lesser extent (Collagen II: $\sim 2.5$, Aggrecan: $\sim 3$, Sox: $\sim 5$ ), in cells cultured in growth medium (Figures $3 \mathrm{c}$ and S9a). These results suggest that the Glc-PA/E-PA and the inducer molecules in chondrogenic differentiation medium may have a synergistic effect on chondrogenic differentiation. It is also remarkable that Glc-PA/E-PA is effective enough to trigger chondrogenesis in the absence of a chondrogenic medium. Chondrogenic differentiation media is considered as a gold standard for the induction of chondrogenesis, and we showed that its synergistic effect with glycopeptide nanofibers have profound effect on chondrogenesis of MSCs. On the other hand, growth media does not have additional growth factors or differentiation inducer molecules, which provides a suitable environment to show the unique scaffold effect of glycopeptide nanofiber system on chondrogenesis without additional growth factor and inducer molecules. This also provides the elimination of shortcomings of using differentiation media such as batch-to-batch variation and expensive cost of growth factors as well as not truly resembling native cartilage tissue environment. The results of both of these experiments successfully showed the advantage of a glycopeptide nanofiber system on chondrogenic differentiation of MSCs in vitro.

The mMSCs on HA/K-PA showed a different pattern of GAG accumulation and Collagen II and Sox 9 expression, both of which increased at day 7 in chondrogenic and growth media (Figures 3c and S9a). At day 14, GAG deposition and Collagen II expression of cells on HA/K-PA were significantly higher than Glc-PA/E-PA and control groups in growth medium (Figure S8b). Moreover, Sox 9 expression also peaked at day 14 for HA/K-PA treated cells in both growth and chondrogenic media (Figures $3 \mathrm{c}$ and S9a). However, aggrecan expression did not show significant upregulation by cells on HA/K-PA throughout the experimental period, which can be attributed to the scaffold-mediated suppression of aggrecan expression (Figure 3c). The overall pattern of sulfated GAG production matches the pattern of chondrogenic marker expression in both Glc-PA/E-PA and HA/K-PA samples, suggesting that signals received from both nanofibrous network types similarly instruct mMSCs for cartilage-like ECM deposition.

Sulfated GAG deposition patterns were further investigated by visualization of GAG distribution on or around cellular aggregations through Safranin-O staining at days 3, 7, and 14 (Figures 3a, S8a and S8c). Gradual increase in staining intensity, in concert with the increase in aggregate sizes, indicated ongoing differentiation process on Glc-PA/E-PA and HA/K-PA through 14 days. We also investigated cartilagespecific protein expressions by immunolocalization with fluorescence-conjugated antibodies on day 14. Extensive staining of collagen II, aggrecan, and sox 9 in cells cultured on Glc-PA/K-PA further proved chondrogenic differentiation, while cells on HA/K-PA, K-PA/E-PA and tissue culture plate 
showed less prominent staining for cartilage marker proteins (Figures $3 \mathrm{~d}$ and $\mathrm{S} 9 \mathrm{~b}$ ).

To study CD 44 interaction and subsequent signaling responsible for the early induction of the mMSCs toward chondrogenesis, we analyzed the changes in the expression of Sox 9, a transcription factor that activates chondrogenesisrelated pathways, following CD44 blockage. On Glc-PA/E-PA nanofibers, the mMSCs treated with anti-CD44 had significantly decreased Sox 9 expression compared to nontreated mMSCs on day 3 in both growth and chondrogenic media (Figures 4 and S10). Similarly, mMSCs on HA/K-PA also displayed decreased Sox 9 expression following anti-CD44 treatment on day 3. However, CD 44 blocking clearly did not affect Sox 9 expression of mMSCs cultured on K-PA/E-PA and tissue culture plate (Figures $4 \mathrm{a}$ and $4 \mathrm{~b}$ ). This finding is consistent with Hyal-1 expression patterns and suggests that the mMSCs recognize functional units of Glc-PA/E-PA in a similar way with $\mathrm{HA} / \mathrm{K}-\mathrm{PA}$, directing differentiation accordingly.

3D cell culture provides a native-like microenvironment that enhances cell-cell and cell-material cross-talking and therefore allows the regulation of cell behavior to a greater extent than $2 \mathrm{D}$ culture conditions. $3 \mathrm{D}$ cell culture analyses were performed on two groups; the Glc-PA/E-PA and K-PA/E-PA gels. Live/ Dead (Invitrogen, Figure S11a) and Alamar Blue (Invitrogen, Figure S11c) assays demonstrated that both nanofiber gels used for $3 \mathrm{D}$ cell culture studies supported the viability of the mMSCs. SEM images suggest that cells in both Glc-PA/E-PA and K-PA/E-PA gels interacted with the material and exhibited a spherical morphology (Figures S11b and S12). Therefore, glycopeptide nanofiber gels can be used to study cellular behavior in a $3 \mathrm{D}$ environment.

To evaluate the regenerative capacity of glycopeptide gels on articular cartilage, we performed the full thickness osteochondral defect treated with microfracture model. In this model, the subchondral bone is bled to allow the recruitment of bone marrow-derived mesenchymal stem cells (MSCs) into the defect site, which initiates the regeneration process. The ability of the glycopeptide nanofiber gels to promote the differentiation of transported MSCs was compared to a clinically utilized formulation of HA (Hyalgan), and a saline-treated group was included as control. As a model animal, white male New Zealand rabbits were used for animal experiments. Defects (1.5 $\mathrm{mm}$ in depth and diameter) were created in the trochlear groove of each knee and treated with Glc-PA/E-PA, Hyalgan, or saline following microfracture. Four animals were used for each study group, and all animals were sacrificed 12 weeks postoperation. Microfracture-treated osteochondral defect model in rabbits is a widely used model for the cartilage regeneration studies, especially to analyze the early stages of therapy. ${ }^{40}$ Due to the fact that the glycopeptide nanofiber system provides early induction of chondrogenesis in vitro, we employed this model to further evaluate the regenerative capacity of this system at early stages of chondrogenic regeneration in vivo. In this model, the healing effect of the glycopeptide nanofiber system was shown to be significantly higher and more efficient than both saline-treated and Hyalgantreated groups.

Consecutive sections from each sample were stained with Safranin-O and counterstained with Fast Green/Hemotoxylin to qualitatively assess the characteristics of regenerated tissue. Newly formed tissue thickness and integration to surrounding tissue, GAG and collagen II-rich matrix deposition, and cellular morphology and density were analyzed in detail for each section. All samples showed full closure of the defect site. No tissue necrosis or infiltration by immune cells was observed in any of the samples. Samples treated with Hyalgan and saline generally exhibited a weak vertical and basal integration to surrounding tissue, with fissures and partial detachment (Figure $5 \mathrm{a}, \mathrm{b})$. Moreover, weak Safranin-O stainings and loose tissue

$$
\text { a } \quad b
$$
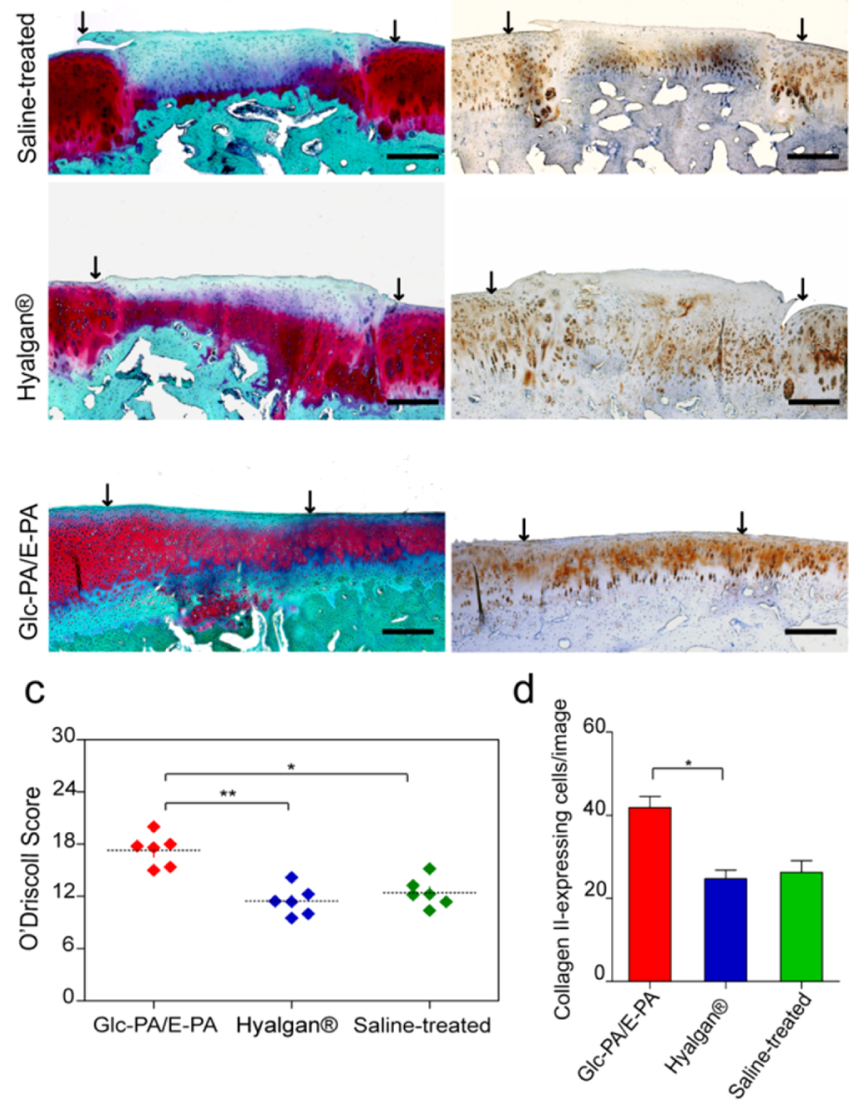

Figure 5. Hyaline cartilage formation predominates in groups treated with glycopeptide nanofiber gels following microfracture. Histological assessment was performed 12 weeks after treatment. Tissue sections stained with Safranin-O (a) and Collagen II immunostain (b). (c) O'Driscoll scoring system was used to evaluate repair tissue characteristics. Each square shape represents a single sample belonging to the relevant treatment group. (d) Quantitative analysis of collagen II expressing cells in the defect sites of each treatment group. Error bars indicate SEM, $n=3, * * p<0.05, * p<0.01$.

arrangements in these groups indicate the presence of fibrous cartilage with apparent surface irregularities, including disruption and delamination. Spherical chondrocytes were distributed in a limited part of the regenerated tissue, and hypocellularity was observed at the site of integration (Figure 5a). Collagen II immunostainings showed that cells in salinetreated groups deposited relatively low amounts of collagen, especially at the uppermost part of the regenerated tissue (Figure 5b).

The nonmatching distributions of collagen II and sGAG in Hyalgan-treated samples indicated the heterogeneous nature of the regenerated tissue (Figure 5a,b). In contrast, defect sites filled with Glc-PA/E-PA nanofiber gel completely integrated to the surrounding tissue at two lateral sites, and basally integrated 
to the subchondral bone. The regenerated tissue was smooth, displayed no evidence of abrasive damage and closely matched the surrounding tissue in appearance. Chondrocytes were generally distributed in columnar structures, as is observed in lacunae, and showed zonal organization (i.e., were spindleshaped in the superficial zone and rounded at the midzone). The strong and evenly distributed staining of collagen II and Safranin-O and relatively low staining of Collagen I (Figure S13) suggests that the regenerated tissue was dominated by hyaline-like cartilage and closely matched the morphology and composition of the surrounding tissue.

The characteristics of the regenerated tissue were also scored for quantitative assessment. A validated 24-point O'Driscoll scoring system optimized for cartilage repair in animal studies was used for this purpose. ${ }^{41}$ This test encompasses four major categories; the nature of the predominant tissue, structural characteristics, cellular evidence of degeneration, and degenerative changes in adjacent cartilage. Results were derived from six animals each for the saline-treated, Hyalgan, and Glc-PA/EPA groups and concluded the following means: $12.55 \pm 6.02$, $11.64 \pm 5.02$, and $17.31 \pm 4.83$, respectively (Figure $5 c$ and Table S2). A higher score and lower standard deviation was achieved in Glc-PA/E-PA-treated groups, while Hyalgantreated groups showed a lower score that was statistically indistinguishable from the saline-treated group. We also investigated the number of cells stained positively with collagen II in regenerated tissues. The ratio of collagen II-positive cells was significantly higher in the Glc-PA/E-PA-treated group, with abundant distribution in the midzone of newly formed tissue, compared to the saline-treated or Hyalgan-treated group (Figures 5d and S14). In vivo experiments overall suggested that Glc-PA/E-PA treatment results in the complete regeneration of the defect site with hyaline-like characteristics within a 12-week period, which provides further support for the capacity of glycopeptide nanofibers for early induction of chondrogenesis.

Mature cartilage tissue is unable to reinitiate its developmental mechanisms after injury and requires exogenous manipulation for functional regeneration. ${ }^{42,43} \mathrm{HA}$ is one of the most prominent regulatory components of mature and developing cartilage extracellular matrix. ${ }^{44}$ In developing cartilage, the HA-rich extracellular matrix regulates the formation of condensation units that will later serve as templates for the cartilage anlagen. ${ }^{3,9,45}$ Moreover, several reports have shown that HA molecules initiate and enhance in vitro chondrogenic differentiation of stem cells, and that CD44 binding is necessary for this process. ${ }^{6,10}$ The modulation of CD44 and its downstream elements provide an effective means for initiating and maintaining the chondrogenic differentiation of mesenchymal stem cells. In this work, we report a supramolecular design of glycopeptide nanofibers, which presents functional chemical units found in the native HA. These nanofibers stimulate early commitment of mMSCs into the chondrogenic lineage through CD44 interactions, thereby replicating the function of native HA networks.

The combination of $\beta$-D-glucose-containing amphiphilic glycopeptide and carboxylic acid-bearing peptide amphiphile molecules allows the imitation of the chemical signature and high charge density of HA. Even though the detailed mechanism of the role of glucose in chondrogenesis is not fully understood, there are studies showing the effect of different glucose-concentration-containing media on MSC chondrogenesis. Previously, low-glucose DMEM increased differentiation of hMSC more than high-glucose media through activation of PKC/TGF- $\beta$ signaling pathway. ${ }^{46}$ However, our system introduces glucose residues on a supramolecular nanofiber scaffold rather than as a soluble factor. Therefore, it more closely resembles native glycosaminoglycans found in natural ECM of the cartilage tissue rather than soluble ligands introduced by cell media or blood glucose in the body.

The GAG-like nanofibrous system morphologically mimics the fibrous extracellular matrix responsible for regulating cell adhesion and protein adsorption processes. A combination of these factors allows the glycopeptide nanofiber matrix to provide a biocompatible and cell-inductive environment for the culturing of mesenchymal stem cells. Our in vitro results suggest that mMSCs recognize glycopeptide nanofibers through HA receptors, and this interaction leads to their early commitment to the chondrogenic lineage. Cellular recognition of Glc-PA/EPA by CD44, as the main adhesion receptor for HA, was shown by in vitro studies. The mMSCs primarily use CD44 for HA binding, and the HA-CD44 interaction has been reported to upregulate the expression of Hyal-1. As such, we tracked Hyal-1 expression of cells following the blocking of CD44 receptors with an anti-CD44 antibody. The downregulation of Hyal-1 expression following CD44 blockade confirmed the possibility that the mMSCs recognize glycopeptide nanofibers in a similar manner to the native HA.

Subsequently, differentiation analysis demonstrated that the differentiation of mMSCs occurs at an earlier stage and is enhanced on glycopeptide nanofibers, even in the absence of chondrogenic factors. The HA promotes chondrogenesis through its interaction with CD44, and the inhibition of this interaction results in downregulation of cartilage related markers. The expression of Sox 9, the master transcription factor of the chondrogenic differentiation pathway, was downregulated in mMSCs cultivated on Glc-PA/E-PA following CD44 blocking, while no difference in expression was observed in K-PA/E-PA control. A similar response to Glc$\mathrm{PA} / \mathrm{E}-\mathrm{PA}$ group was observed in cells grown on $\mathrm{HA} / \mathrm{K}-\mathrm{PA}$ following CD44 blockage, suggesting that the biochemical compositions of Glc-PA/E-PA and HA/K-PA are similar. As such, the Glc-PA/E-PA nanofiber is capable of mimicking the function of HA through its chemical composition, which elicits cellular responses similar to the native HA molecules through CD44 signaling pathways.

Our in vivo results also supported these in vitro findings and suggested that hyaline cartilage formation is enhanced by GlcPA/E-PA treatment in a microfracture-treated cartilage defect model. In addition to the biochemical cues provided by the hydrogel, the structure of the Glc-PA/E-PA matrix itself may assist in maintaining a high concentration of cells at the defect site by facilitating the adhesion of MSCs transferred from bone marrow during microfracture. Moreover, Glc-PA/E-PA treatment may stabilize blood clots and promote the early mechanical stability of the defect site.

\section{CONCLUSION}

In conclusion, we showed that a molecularly designed supramolecular mimic of HA can interact with mesenchymal stem cells through CD 44 receptor and facilitate their commitment to the chondrogenic lineage without the need for exogenous growth factors. A key aspect of the current design is that it can be used in place of naturally derived HA to eliminate the potential health hazards associated with natural scaffolds. Our in vivo results also suggest that glycopeptide 
nanofibers can be used for less invasive and cell-free in situ cartilage regeneration approaches by inducing the chondrogenesis of mesenchymal stem cells released from bone marrow following microfracture.

\section{ASSOCIATED CONTENT}

\section{S Supporting Information}

The Supporting Information is available free of charge on the ACS Publications website at DOI: 10.1021/acs.biomac.5b01669.

Information regarding SAXS data analysis and additional figures and tables as described in the text (PDF)

\section{AUTHOR INFORMATION}

\section{Corresponding Authors}

*E-mail address: moguler@unam.bilkent.edu.tr (M.O.G.).

*E-mail address: atekinay@unam.bilkent.edu.tr (A.B.T.).

\section{Author Contributions}

${ }^{\perp}$ S.U.Y. and M.S.E. contributed equally to this manuscript.

\section{Notes}

The authors declare no competing financial interest.

\section{ACKNOWLEDGMENTS}

This work was supported by TUBITAK Grants 113 T045 and 114S913. A.B.T. acknowledges support from the Turkish Academy of Sciences Distinguished Young Scientist Award (TUBA-GEBIP). S.U.Y., M.S.E., and E.A. are supported by TUBITAK BIDEB PhD fellowship. We would like to thank $\mathrm{Z}$. Erdogan and M. Guler for their technical help in LC-MS studies and TEM imaging, respectively.

\section{REFERENCES}

(1) Bhosale, A. M.; Richardson, J. B. Articular cartilage: structure, injuries and review of management. Br. Med. Bull. 2008, 87 (1), 7795.

(2) Hunziker, E. Articular cartilage repair: basic science and clinical progress. A review of the current status and prospects. Osteoarthr. Cartil. 2002, 10 (6), 432-463.

(3) Hall, B. K.; Miyake, T. Divide, accumulate, differentiate: cell condensation in skeletal development revisited. Int. J. Dev. Biol. 1995, $39,881-893$.

(4) Mithoefer, K.; Williams, R. J.; Warren, R. F.; Potter, H. G.; Spock, C. R.; Jones, E. C.; Wickiewicz, T. L.; Marx, R. G. The microfracture technique for the treatment of articular cartilage lesions in the knee. J. Bone Joint Surg. Am. 2005, 87 (9), 1911-1920.

(5) Mithoefer, K.; McAdams, T.; Williams, R. J.; Kreuz, P. C.; Mandelbaum, B. R. Clinical efficacy of the microfracture technique for articular cartilage repair in the knee an evidence-based systematic analysis. Am. J. Sports Med. 2009, 37 (10), 2053-2063.

(6) Bian, L.; Guvendiren, M.; Mauck, R. L.; Burdick, J. A. Hydrogels that mimic developmentally relevant matrix and $\mathrm{N}$-cadherin interactions enhance MSC chondrogenesis. Proc. Natl. Acad. Sci. U. S. A. 2013, 110 (25), 10117-10122.

(7) Toh, W. S.; Lim, T. C.; Kurisawa, M.; Spector, M. Modulation of mesenchymal stem cell chondrogenesis in a tunable hyaluronic acid hydrogel microenvironment. Biomaterials 2012, 33 (15), 3835-3845.

(8) Kim, I. L.; Mauck, R. L.; Burdick, J. A. Hydrogel design for cartilage tissue engineering: a case study with hyaluronic acid. Biomaterials 2011, 32 (34), 8771-8782.

(9) Knudson, C. B. Hyaluronan and CD44: strategic players for cellmatrix interactions during chondrogenesis and matrix assembly. Birth Defects Res., Part C 2003, 69 (2), 174-196.

(10) Wu, S.-C.; Chen, C.-H.; Chang, J.-K.; Fu, Y.-C.; Wang, C.-K.; Eswaramoorthy, R.; Lin, Y.-S.; Wang, Y.-H.; Lin, S.-Y.; Wang, G.-J.;
Ho, M.-L. Hyaluronan initiates chondrogenesis mainly via CD44 in human adipose-derived stem cells. J. Appl. Physiol. 2013, 114 (11), 1610-1618.

(11) Drury, J. L.; Mooney, D. J. Hydrogels for tissue engineering: scaffold design variables and applications. Biomaterials 2003, 24 (24), 4337-4351.

(12) Lee, K. Y.; Mooney, D. J. Hydrogels for tissue engineering. Chem. Rev. 2001, 101 (7), 1869-1880.

(13) Lutolf, M.; Hubbell, J. Synthetic biomaterials as instructive extracellular microenvironments for morphogenesis in tissue engineering. Nat. Biotechnol. 2005, 23 (1), 47-55.

(14) Fakhari, A.; Berkland, C. Applications and emerging trends of hyaluronic acid in tissue engineering, as a dermal filler and in osteoarthritis treatment. Acta Biomater. 2013, 9 (7), 7081-7092.

(15) Weyers, A.; Linhardt, R. J. Neoproteoglycans in tissue engineering. FEBS J. 2013, 280 (10), 2511-2522.

(16) Xu, X.-D.; Liang, L.; Cheng, H.; Wang, X.-H.; Jiang, F.-G.; Zhuo, R.-X.; Zhang, X.-Z. Construction of therapeutic glycopeptide hydrogel as a new substitute for antiproliferative drugs to inhibit postoperative scarring formation. J. Mater. Chem. 2012, 22 (35), 18164-18171.

(17) Zhao, F.; Weitzel, C. S.; Gao, Y.; Browdy, H. M.; Shi, J.; Lin, H.C.; Lovett, S. T.; Xu, B. $\beta$-Galactosidase-instructed formation of molecular nanofibers and a hydrogel. Nanoscale 2011, 3 (7), 28592861.

(18) Zhao, F.; Heesters, B. A.; Chiu, I.; Gao, Y.; Shi, J.; Zhou, N.; Carroll, M. C.; Xu, B. L-Rhamnose-containing supramolecular nanofibrils as potential immunosuppressive materials. Org. Biomol. Chem. 2014, 12 (35), 6816-6819.

(19) Roytman, R.; Adler-Abramovich, L.; Kumar, K. A.; Kuan, T.-C.; Lin, C.-C.; Gazit, E.; Brik, A. Exploring the self-assembly of glycopeptides using a diphenylalanine scaffold. Org. Biomol. Chem. 2011, 9 (16), 5755-5761.

(20) Bonduelle, C.; Lecommandoux, S. Synthetic Glycopolypeptides as Biomimetic Analogues of Natural Glycoproteins. Biomacromolecules 2013, 14 (9), 2973-2983.

(21) Ren, K.; He, C.; Xiao, C.; Li, G.; Chen, X. Injectable glycopolypeptide hydrogels as biomimetic scaffolds for cartilage tissue engineering. Biomaterials 2015, 51, 238-249.

(22) Cui, H.; Webber, M. J.; Stupp, S. I. Self-assembly of peptide amphiphiles: From molecules to nanostructures to biomaterials. Biopolymers 2010, 94 (1), 1-18.

(23) Toksoz, S.; Acar, H.; Guler, M. O. Self-assembled onedimensional soft nanostructures. Soft Matter 2010, 6 (23), 5839-5849.

(24) Hartgerink, J. D.; Beniash, E.; Stupp, S. I. Self-assembly and mineralization of peptide-amphiphile nanofibers. Science 2001, 294 (5547), 1684-1688.

(25) Capito, R. M.; Azevedo, H. S.; Velichko, Y. S.; Mata, A.; Stupp, S. I. Self-assembly of large and small molecules into hierarchically ordered sacs and membranes. Science 2008, 319 (5871), 1812-1816.

(26) Arslan, E.; Garip, I. C.; Gulseren, G.; Tekinay, A. B.; Guler, M. O. Bioactive supramolecular peptide nanofibers for regenerative medicine. Adv. Healthcare Mater. 2014, 3 (9), 1357-1376.

(27) Shah, R. N.; Shah, N. A.; Del Rosario Lim, M. M.; Hsieh, C.; Nuber, G.; Stupp, S. I. Supramolecular design of self-assembling nanofibers for cartilage regeneration. Proc. Natl. Acad. Sci. U. S. A. 2010, 107 (8), 3293-3298.

(28) Ustun, S.; Tombuloglu, A.; Kilinc, M.; Guler, M. O.; Tekinay, A. B. Growth and differentiation of prechondrogenic cells on bioactive self-assembled peptide nanofibers. Biomacromolecules 2013, 14 (1), $17-26$.

(29) Mammadov, R.; Mammadov, B.; Toksoz, S.; Aydin, B.; Yagci, R.; Tekinay, A. B.; Guler, M. O. Heparin mimetic peptide nanofibers promote angiogenesis. Biomacromolecules 2011, 12 (10), 3508-3519.

(30) Boekhoven, J.; Stupp, S. I. 25th anniversary article: supramolecular materials for regenerative medicine. Adv. Mater. 2014, 26 (11), 1642-1659.

(31) Farndale, R. W.; Buttle, D. J.; Barrett, A. J. Improved quantitation and discrimination of sulphated glycosaminoglycans by 
use of dimethylmethylene blue. Biochim. Biophys. Acta, Gen. Subj. 1986, 883 (2), 173-177.

(32) Liu, M.; Barany, G.; Live, D. Parallel solid-phase synthesis of mucin-like glycopeptides. Carbohydr. Res. 2005, 340 (13), 2111-2122.

(33) Dehsorkhi, A.; Castelletto, V.; Hamley, I. W. Self-assembling amphiphilic peptides. J. Pept. Sci. 2014, 20 (7), 453-467.

(34) Pedersen, J. S.; Schurtenberger, P. Scattering functions of semiflexible polymers with and without excluded volume effects. Macromolecules 1996, 29 (23), 7602-7612.

(35) Chen, W.-R.; Butler, P. D.; Magid, L. J. Incorporating intermicellar interactions in the fitting of SANS data from cationic wormlike micelles. Langmuir 2006, 22 (15), 6539-6548.

(36) Chen, T.; Hilton, M. J.; Brown, E. B.; Zuscik, M. J.; Awad, H. A. Engineering superficial zone features in tissue engineered cartilage. Biotechnol. Bioeng. 2013, 110 (5), 1476-1486.

(37) Nicoll, S. B.; Barak, O.; Csóka, A. B.; Bhatnagar, R. S.; Stern, R. Hyaluronidases and CD44 undergo differential modulation during chondrogenesis. Biochem. Biophys. Res. Commun. 2002, 292 (4), 819825 .

(38) Harada, H.; Takahashi, M. CD44-dependent intracellular and extracellular catabolism of hyaluronic acid by hyaluronidase-1 and-2. J. Biol. Chem. 2007, 282 (8), 5597-5607.

(39) Johnstone, B.; Hering, T. M.; Caplan, A. I.; Goldberg, V. M.; Yoo, J. U. In vitrochondrogenesis of bone marrow-derived mesenchymal progenitor cells. Exp. Cell Res. 1998, 238 (1), 265-272.

(40) Chu, C. R.; Szczodry, M.; Bruno, S. Animal models for cartilage regeneration and repair. Tissue Eng., Part B 2010, 16 (1), 105-115.

(41) O’Driscoll, S. W.; Keeley, F. W.; Salter, R. The chondrogenic potential of free autogenous periosteal grafts for biological resurfacing of major full-thickness defects in joint surfaces under the influence of continuous passive motion. An experimental investigation in the rabbit. J. Bone Joint Surg. Am. 1986, 68 (7), 1017-1035.

(42) Makris, E. A.; Gomoll, A. H.; Malizos, K. N.; Hu, J. C.; Athanasiou, K. A. Repair and tissue engineering techniques for articular cartilage. Nat. Rev. Rheumatol. 2014, 11, 21-34.

(43) Athanasiou, K. A.; Darling, E. M.; Hu, J. C. Articular cartilage tissue engineering. Synthesis Lectures on Tissue Engineering 2009, 1 (1), $1-182$.

(44) Ishida, O.; Tanaka, Y.; Morimoto, I.; Takigawa, M.; Eto, S. Chondrocytes are regulated by cellular adhesion through CD44 and hyaluronic acid pathway. J. Bone Miner. Res. 1997, 12 (10), 16571663.

(45) Knudson, C. B.; Knudson, W. Cartilage Proteoglycans. Semin. Cell Dev. Biol. 2001, 12, 69-78.

(46) Tsai, T. L.; Manner, P. A.; Li, W. J. Regulation of mesenchymal stem cell chondrogenesis by glucose through protein kinase C/ transforming growth factor signaling. Osteoarthr. Cartil. 2013, 21 (2), 368-376. 